\title{
CAVERNOMA GIGANTE
}

\section{Relato de dois casos}

\author{
Guilherme Cabral de Andrade1, Mirto Nelso Prandini², Fernando Menezes Braga ${ }^{3}$
}

\begin{abstract}
RESUMO - Angiomas cavernosos ou hemangiomas ou ainda cavernomas são malformações do sistema nervoso central, classificadas como lesões cerebrais vasculares ocultas, frequentemente assintomáticas, sendo relativamente raras. Definidas histologicamente como massas compactas cavernosas ou canais sinusoidais de vários tamanhos, com paredes finas no interior do parênquima cerebral e sem intervenção do tecido glial. Podem ocorrer em qualquer faixa etária inclusive em neonatos. Na maioria das vezes são lesões de tamanho reduzido, localizadas no interior do parênquima encefálico. Angiograficamente não mostram alterações com circulação patológica, podendo mesmo não ser diagnosticados pela tomografia, sendo o exame ideal para o seu diagnóstico a ressonância magnética de crânio. Os cavernomas são lesões histologicamente benignas mas, dependendo de sua localização, podem trazer grandes transtornos neurológicos e ser irressecáveis. A exérese cirúrgica da lesão é o tratamento de escolha se a lesão for única e em localização favorável e estiver desencadeando sintomatologia neurológica prejudicial ao paciente. Apresentamos dois casos de cavernomas gigantes que apresentaram boa evolução após ressecção cirúrgica completa. Descritos como gigantes, foram encontrados apenas três casos individuais na literatura .
\end{abstract}

PALAVRAS-CHAVES: cavernoma, angioma cavernoso gigante.

\begin{abstract}
Giant cavernous angioma: report of two cases
ABSTRACT - Cavernous angiomas or haemangiomas or yet cavernomas are malformations of the central nervous system classified as occult vascular brain lesions. These rare lesions are clinically silent. They are defined by the presence of abnormally large vascular cavities or sinusoids channels of variable size, with sharp walls, located inside but not invading the brain parenchyma. They can occur at any age, including the neonatal period. Most of the small lesions are located inside the brain parenchyma. No abnormal circulation can be demonstrated in angiography and CT scan can be helpful for diagnosis only in rare occasions. Magnetic resonance is the best exam to demonstrate the lesion. Despite the benign character some lesions may cause neurologic dysfunction when their removal may be difficult. Complete extirpation is the best treatment if the lesion is favorable located and is causing neurological dysfunction. Two cases of giant cavernomas with good outcome after total removal are present. Only three cases of giant cavernomas were reported in the literature.
\end{abstract}

KEY WORDS: cavernoma, giant cavernous angioma.

Cavernomas intracerebrais são malformações vasculares raras, angiograficamente ocultas ${ }^{1}$, com características histopatológicas próprias e distintas de outras malformações vasculares cerebrais (Tabela 1$)^{2}$. De acordo com Russel e Rubinstein ${ }^{3}, 75 \%$ são supratentoriais, podendo acometer qualquer lobo do cérebro. Apresentam localização preferencial na região da fissura rolândica e são duas vezes mais frequentes no hemisfério direito em comparação com o esquerdo. Quando infratentoriais, a localização mais comum é no tronco cerebral (ponte). Outras locali- zações mais raras estão no cerebelo ${ }^{4}$, medula espinhal $^{5}$, tentório ${ }^{6}$, região do clívus ${ }^{7}$, extra axiais envolvendo os seios durais ${ }^{8}$, intraventriculares ${ }^{9,10}$, em região da pineal ${ }^{11}$, na duramáter ${ }^{12}$, na calota craniana ${ }^{13}$. Podem apresentar calcificações ${ }^{14}$, ser múltiplos, classificados como hemangiomatose ${ }^{15}$ e múltiplos com calcificações ${ }^{16}$. Podem também apresentar-se associados a neoplasias gliais ${ }^{17}$. Não há predomínio por sexo $^{18}$. Compreendem cerca de 5 a $13 \%$ das lesões vasculares do sistema nervoso central ${ }^{19}$ e 0,02 a $0,13 \%$ dos casos, em amplas séries de necropsia ${ }^{20}$.

Disciplina de Neurocirurgia da Universidade Federal de São Paulo, Escola Paulista de Medicina, São Paulo SP, Brasil: ${ }^{1}$ Neurocirurgião estagiário do Setor de neurocirurgia vascular; ${ }^{2}$ Docente; ${ }^{3}$ Professor Titular

Recebido 27 Agosto 2001, recebido na forma final 11 Dezembro 2001. Aceito 14 Dezembro 2001. 
Tabela 1. Critérios histológicos para classificação das malformações vasculares (MAV) do cérebro.

\section{MAV'S}

Conjunto de artérias e veias anormais; paredes dos vasos contendo elastina e músc. liso tipicamente espessados (artérias hipertrofiadas e veias arterializadas); invasão mínima de tec gliótico.

\section{ANGIOMAS CAVERNOSOS}

Conjunto de canais (cavernas) com paredes finas e desprovidas de elastina e músc. liso; cavernas contendo sangue e/ou trombos em variados estágios de organização; sem interação com tec. cerebral exceto na periferia da lesão.

\section{ANGIOMAS VENOSOS}

Coleções de pequenas veias geralmente arranjadas em torno de uma ou mais veias maiores; histologicamente possuem parede venosa típica; interação normal com tec.gliótico.

\section{MALFORMAÇÕES CAPILARES}

Coleções de capilares dilatados; interação normal com tec. gliótico.

\section{MALFORMAÇÕES MISTAS}

Ocorre combinação de duas ou mais malformações distintas histologicamente em regiões separadas da mesma lesão.

Cavernomas podem ser esporádicos ou familiares. Os casos familiares são comuns na população hispanoamericana, têm caráter autossômico dominante e, frequentemente, são múltiplos ${ }^{15}$. Os cavernomas são comumente observados entre a $3^{\mathrm{a}}$ e $5^{\mathrm{a}}$ décadas, mas podem ser diagnosticados em qualquer faixa etária, sendo mais raros na infância ${ }^{21}$. Cavernomas gigantes são ainda mais raros. Encontramos apenas três casos descritos na literatura ${ }^{22-24}$. Relatamos dois casos.

\section{CASOS}

Caso1. Menina de 7 anos (RG-HSP 730.370), admitida em 02/01/1991, com história de macrocrania desde o nascimento, sem qualquer outra manifestação clínica. No exame apresentava macrocrania com $+/-55 \mathrm{~cm}$ de perímetro craniano $(\mathrm{PC})$ e sem qualquer outra alteração neurológica. A tomografia computadorizada (TC) de crânio com e sem contraste mostrou grande massa tumoral fronto parietal esquerda, hiperdensa e hipercaptante, de limites regulares, medindo $14 \times 12 \mathrm{~cm}$, com área hipodensa central (Fig 1). Craniotomia ampla fronto parietal esquerda, após abertura da dura máter, mostrou grande lesão expansiva na região fronto parietal (Fig 2). Durante a manipulação cirúrgica observou-se que a lesão, possuía coloração vinhosa, e continha em seu interior pequenos cistos contendo líquido escuro. Realizou-se ressecção total da lesão (Fig 3). A paciente teve evolução favorável, sem intercorrências, retornando a escola em 20 dias em boas condições físicas e mentais. $\mathrm{O}$ exame histopatológico evidenciou um cavernoma, com tecido composto por sinu- sóides com paredes finas, desprovidas de musculatura lisa e elastina, com sangue trombosado em seu interior e sem relação com tecido glial normal. A investigação radiológica pós operatória não revelou lesão residual (Fig 4). No seguimento de 4 anos, a paciente apresentava-se com bom desenvolvimento neuropsicomotor, sem qualquer limitação de suas atividades.

Caso 2. Menina de 7 meses, nascida de parto cesáreo em 11/1990. Evoluiu sem intercorrências até os 4 meses de idade, quando apresentou episódio convulsivo tônicoclônico generalizado, na ausência de febre, sendo medicada com fenobarbital $20 \mathrm{mg} / \mathrm{dia}$. Dois meses após, aos 6 meses de idade, apresentou novo episódio convulsivo, semelhante ao anterior. Reavaliada percebeu-se aumento do PC, com tensão da fontanela bregmática. $O$ exame neurológico mostrava uma criança apática. A TC de crânio, evidenciou grande processo expansivo tumoral, na região fronto parietal esquerda, hiperdenso, hipercaptante e de característica cística, com importante efeito de massa. Submetida à ressonância magnética (RM) de crânio, evidenciou-se grande lesão expansiva, cística, com hipersinal em T2 (Fig 5 ) na região fronto parietal esquerda. Com a hipótese diagnóstica de cavernoma gigante, foi submetida a extensa craniotomia fronto têmporo parietal esquerda em 05/1991 e, após a abertura da dura máter, observou-se lesão cística, no interior do parênquima encefálico, bastante tensa, com drenagem de líquido xantocrômico vinhoso espesso após a abertura da membrana. Foi feita exérese total da lesão, confirmada com a realização de TC pós operatória (Fig 6). No período pós operatório imediato apresentava discreta monoparesia braquial direita, que

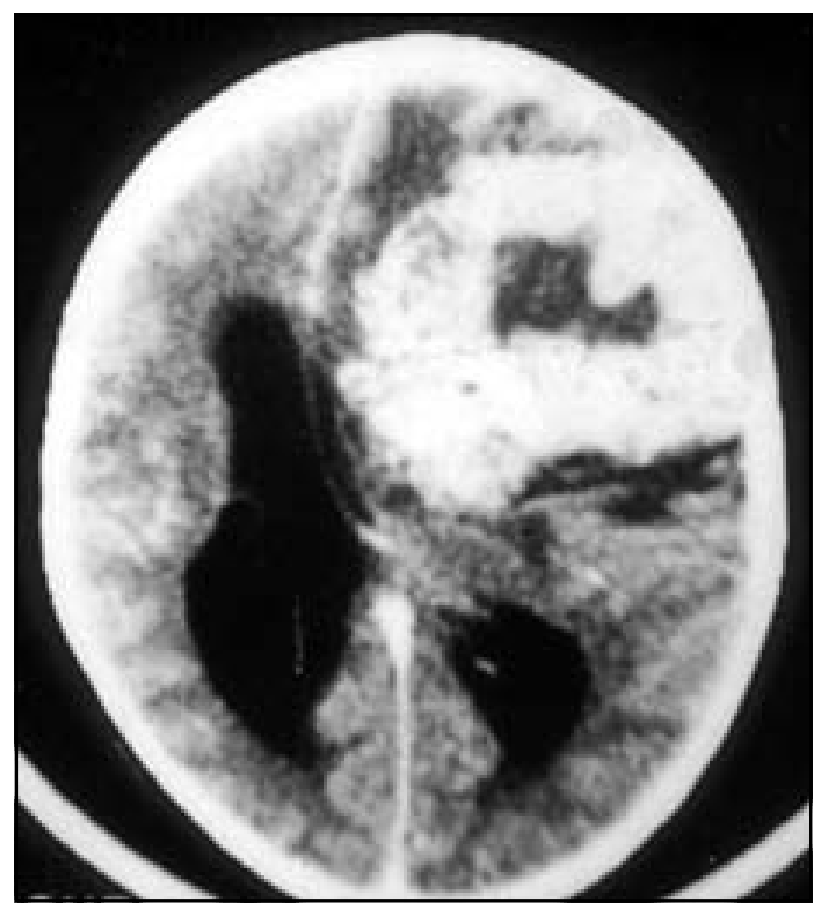

Fig 1. Caso 1, CT com contraste pré operatório, cavernoma gigante FP esquerdo. 

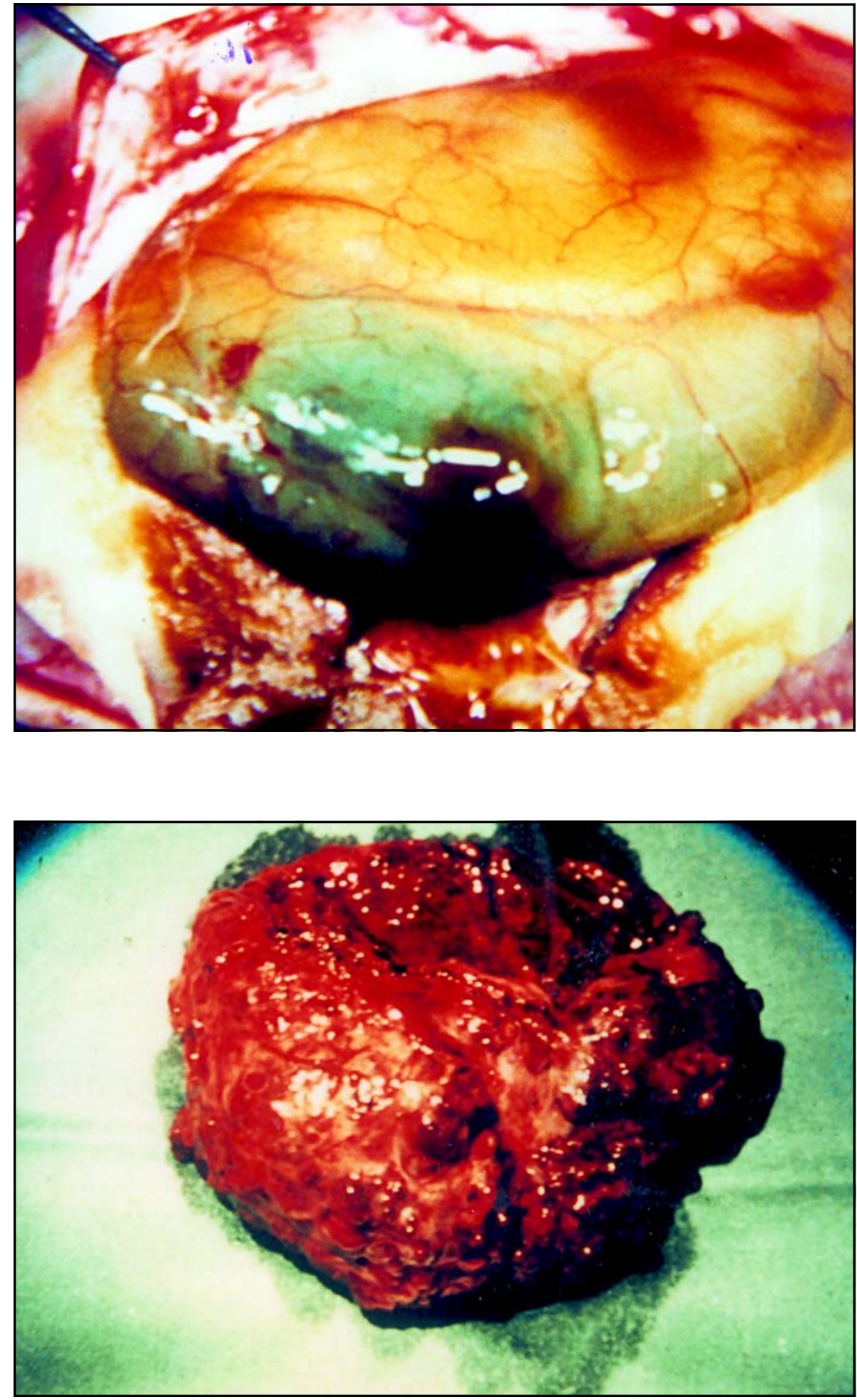

Fig 2. Caso 1, visão intra operatória de grande lesão expansiva FP esquerda.
Fig 3. Caso 1, foto de peça cirugica medindo $14 \times 12 \mathrm{~cm}$, com aspecto de TEC hepático. regrediu em 3 meses. Em um seguimento de 5 anos a paciente não apresentou qualquer intercorrência.

\section{DISCUSSÃO}

Os cavernomas, hemangiomas cavernosos ou angiomas cavernosos são malformações vasculares cerebrais do grupo das malformações angiografi- camente ocultas ${ }^{8}$. Possuem caráter esporádico ou familiar. Quando familiar são mais comumente diagnosticados na população hispano-americana, com caráter autossômico dominante e geralmente múltiplos, o primeiro gene (CCM1) sendo mapeado no braço longo do cromossomo 7 (7q11.2q21) ${ }^{25,26}$ e outros dois no braço curto do cromossomo 7 (7p15- 


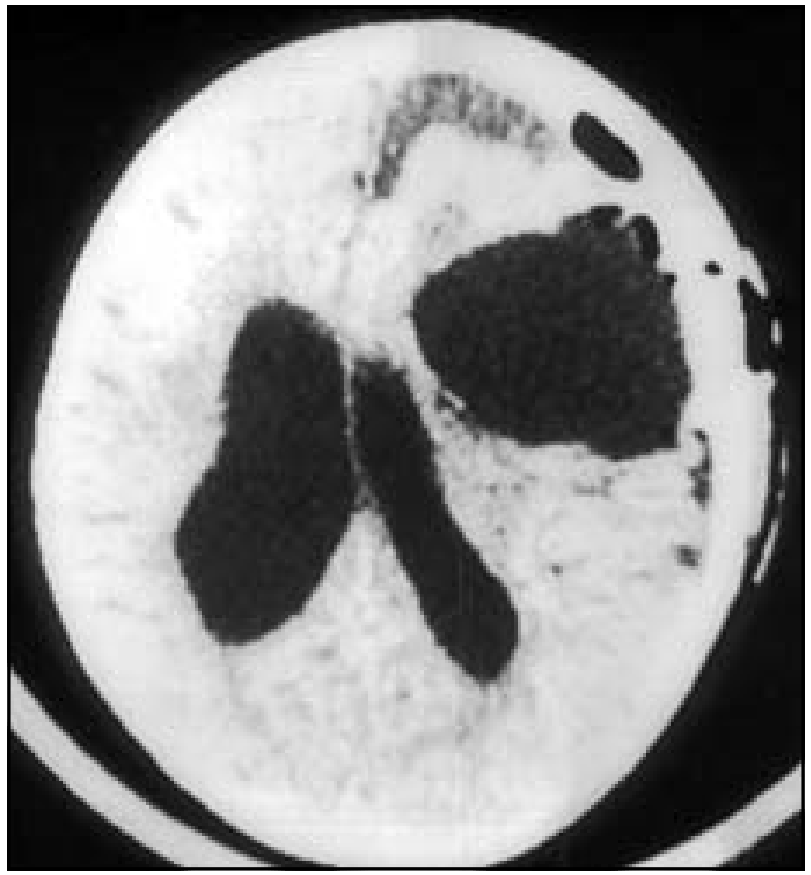

Fig 4. Caso 1, CT controle pós operatório com exérese total da lesão.

13) e no braço longo do cromossomo 3 (3q25.227) ${ }^{27}$. Devido a algumas de suas características como tamanho, lesões múltiplas e localização podem, apesar de serem lesões benignas, apresentar risco elevado de morbidade ${ }^{18}$. Podem variar de alguns milímetros a vários centímetros de diâmetro. Podem apresentar calcificação e ocasionalmente cistos. Apresentam localização supratentorial (75\%) nos mais variados sítios. Descritos como cavernomas gigantes encontramos na literatura três casos (Tabela 2), porém não há definição na literatura no que diz respeito ao tamanho dessas lesões.

Os cavernomas podem acometer qualquer faixa etária, mais comumente entre a $3^{a}$ e $5^{a}$ décadas, sendo menos encontrados nas faixas etárias extremas $^{3}$, sendo também considerados congênitos. Segundo Arnstein e cols. ${ }^{28}$ que descrevem cavernoma em um recém-nascido de 3 dias de idade, tumores sintomáticos antes da idade dos 60 dias podem ser considerados congênitos. Clinicamente, apresentamse com convulsões, cefaléia, acidente vascular cerebral hemorrágico e déficit focal, por ordem de frequência. Nos dois casos já descritos como gigantes em crianças, juntamente com os dois apresentados neste estudo, aumento do perímetro cefálico foi uma manifestação comum.

A angiografia e a TC podem ser negativas em até $1 / 3$ dos casos de cavernomas. A RM de crânio é o exame neurorradiológico de escolha e de maior acurácia no diagnóstico destas lesões. Nos casos con-

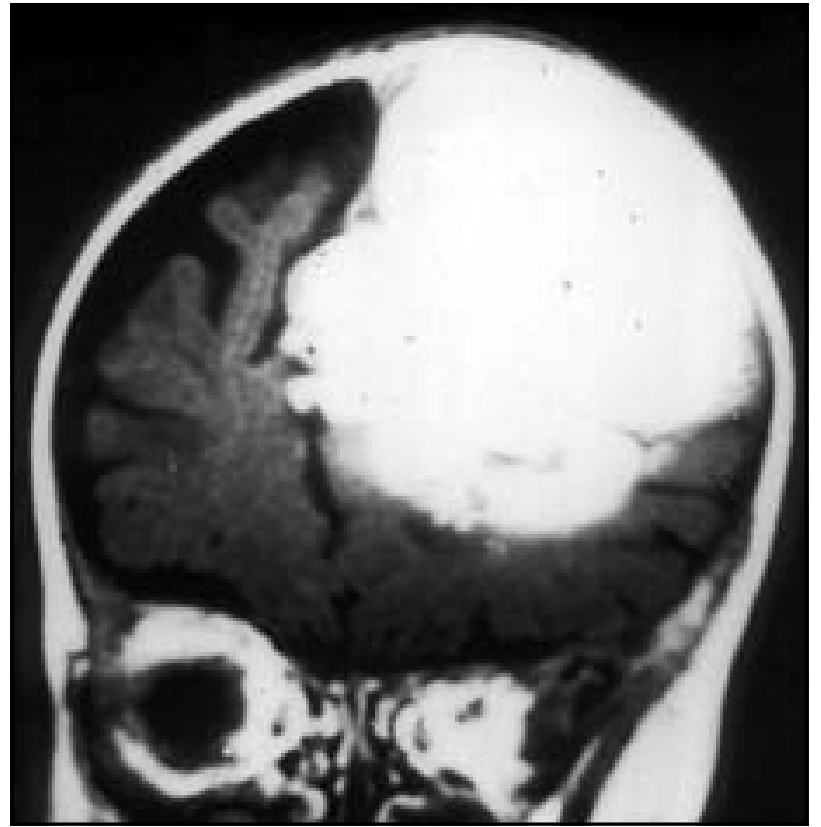

Fig 5. Caso 2, Rm mostrando grande lesão exansiva e cística FP esquerdo com hipersinal em T2.

siderados como cavernomas gigantes, a TC identifica a lesão porém há a necessidade de realização da RM, em virtude de ser um exame de maior acurácia, sendo importante na delimitação da lesão e no estudo das suas relações com o parênquima normal, fatos importantes para um melhor planejamento cirúrgico. $\mathrm{O}$ exame mostrou em ambas, grande lesão tumoral de caráter expansivo e cístico, localizada na

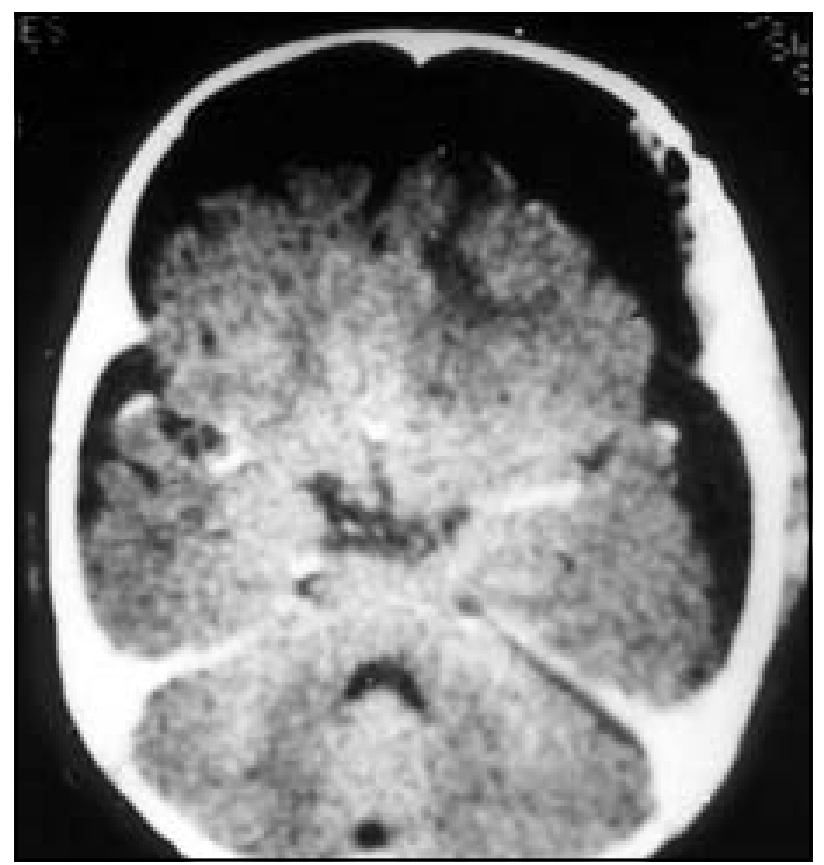

Fig 6. Caso 2, CT controle pós opreratório monstrando exérese completa da lesão 
Tabela 2. Casos de cavernomas gigantes publicados entre 1966 e 2001.

\begin{tabular}{|c|c|c|c|c|c|c|c|}
\hline Autor & Ano & Idade & Sexo & Topografia & Clínica & Cirurgia & Evolução \\
\hline Sansone e col. & 1980 & 72 anos & Fem & $\begin{array}{l}\text { Região } \\
\text { pituitária }\end{array}$ & Diplopia & Não & $\begin{array}{c}\text { Óbito por } \\
\text { adenocarcinoma } \\
\text { de mama }\end{array}$ \\
\hline Chen e Kuo & 1984 & 3,5 meses & Fem & $\begin{array}{c}\text { Fronto } \\
\text { parietal direita }\end{array}$ & $\begin{array}{l}\text { Aumento do } \\
\text { PC/ dist. da } \\
\text { consciência/ } \\
\text { crise convulsiva }\end{array}$ & Não & $\begin{array}{l}\text { Óbito por } \\
\text { hipertensão } \\
\text { intercraniana }\end{array}$ \\
\hline Khosla e col. & 1984 & 3 anos & Fem & $\begin{array}{c}\text { Fronto } \\
\text { parietal direita }\end{array}$ & $\begin{array}{l}\text { Aumento do } \\
\text { PC/dificuldade } \\
\text { para andar }\end{array}$ & $\begin{array}{l}\text { Extirpação } \\
\text { Completa }\end{array}$ & $\begin{array}{c}\text { Boa / sem } \\
\text { intercorrências }\end{array}$ \\
\hline
\end{tabular}

região fronto parietal esquerda. Apenas a segunda paciente realizou também RM de crânio. Por serem lesões que podem não aumentar de tamanho ou, também, não evoluir com sangramento, muitas vezes são diagnosticadas incidentalmente ou no momento da necropsia. Em uma pequena minoria dos casos, alguns fatores podem servir como diagnóstico presuntivo, como a presença de casos familiares e a presença de angiomas cavernosos cutâneos ou em outros órgão ou tecidos ${ }^{2}$.

O tratamento de escolha para as lesões solitárias ou de localização favorável é a ressecção cirúrgica completa da lesão. Lesões múltiplas não possuem tratamento específico. Nas lesões de difícil acesso e quando há duas ou mais hemorragias, pode-se usar como tratamento coadjuvante a radiocirurgia, que oferece bons resultados no que diz respeito à redução do seu tamanho ${ }^{29}$.

Em conclusão, os cavernomas são raros, principalmente quando se apresentam nos extremos das faixas etárias (crianças e idosos) e particularmente quando de grandes dimensões (gigantes). Descrevemos dois casos de cavernomas gigantes diagnosticados em uma criança de 7 anos e em um bêbê de 7 meses. A revisão da literatura mostrou dois relatos em crianças de 3,5 meses e de 7 anos, ao lado de uma descrição de cavernoma gigante em um idoso de 72 anos. Nas 4 crianças a manifestação clínica foi macrocrania. Dos 3 casos descritos na literatura, em apenas um foi realizada a ressecção cirúrgica, com bom resultado. Os outros dois faleceram, sem tratamento cirúrgico.

Os nossos dois casos foram tratados cirurgicamente, com extirpação completa dos cavernomas mostrando excelente evolução. Em crianças que apresentam aumento do PC e em que a TC mostre a presença de grande lesão expansiva de características císticas, deve-se concluir a investigação com RM de crânio e acrescentar ao diagnóstico diferencial o cavernoma. Deve-se submeter o paciente a ressecção cirúrgica com exérese total da lesão, visto que é uma lesão de características benignas, com bom plano de clivagem com o tecido glial normal, sem que ocorram lesões importantes do tecido cerebral normal, com o que os pacientes apresentam boa evolução pós operatória.

\section{REFERÊNCIAS}

1. Robinson JR Jr, Awad IA, Masaryk TJ, Estes ML. Pathological heterogeneity of angiographically occult vascular malformations of the brain. Neurosurgery 1993;33:547-554.

2. Giombini S, Morello G. Cavernous angiomas of the brain, account of fourteen personal cases and review of the literature. Acta Neurochirur 1978;40: 61-82.

3. Russel DS, Rubinstein LJ. Pathology of tumors of the nervous system. 5.Ed. London: Edward Arnold, 1989:730-736.

4. McCormick WF, Hardman JM, Boulter TR. Vascular malformations ("angiomas") of the brain, with special reference to those occuring in the posterior fossa. J Neurosurg 1985;28:241-251.

5. Zevgaridis D, Medele RJ, Hamburger C, Steiger HJ, Reulen HJ. Cavernous haemangiomas of the spinal cord: a review of 117 cases. Acta Neurochirur(Wien) 1999;141:237-245.

6. Quattrocchi KB, Kissel P, Ellis WG, Frank EH. Cavernous angioma of the tentorium cerebelli, case report. J Neurosurg 1989;71:935-937.

7. Tashiro T, Inoue Y, Nemoto $Y$, et al. Cavernous hemangioma of the clivus: case report and review of the literature. AJNR 1991;12:1193-1194.

8. Meyer FB, Lombardi D, Scheithauer B, Nichols DA. Extra-axial cavernous hemangiomas involving the dural sinuses. J Neurosurg 1990;73:187-192.

9. Chadduck WM, Binet EF, Farrell FW Jr, Araoz CA, Reding DL. Intraventricular cavernous hemangioma: report of three cases and review of the literature. Neurosurgery 1985;16:189-197.

10. Iwasa H, Indei I, Sato F. Intraventricular cavernous hemangioma: case report. J Neurosurg 1983;59: 153-157.

11. Lombardi D, Scheithaeur BW, Villani RM, Giovanelli M, Tribolet N. Cavernous haemangioma of the pineal region. Acta Neurochir(Wien) 1996; 138:678-683.

12. Saldaña CJ, Zimman H, Alonso P, Mata PR. Neonatal cavernous hemangioma of the dura mater: case report. Neurosurgery 1991;29:602-605.

13. Bizzozero L, Talamonti CS, Villa F, Brusamolino R, Collice M. Cavernous hemangioma of the skull: case report and review of the literature. J Neurosurg Sci 1997;41:419-421.

14. Moritake K, Handa H, Nozaki K, Tomiwa K. Tentorial cavernous angioma with calcification in a neonate. Neurosurgery 1985;16;207-211. 
15. Labauge P, Brunereau L, Lévy C, Laberge S, Houtteville JP. The natural history of familial cerebral cavernomas: a retrospective MRI study of 40 patients. Neuroradiology 2000;42:327-332.

16. Tateno A, Matsui A, Sakuragawa N, Arima M. Two siblings with multiple intracranial haemangiomatosis with calcification. J Neurol 1985; 232:112-114

17. Edwin G, Sotrel A, Welch K. Cerebral hemangioma with glial neoplasia (angioglioma?): report of two cases. J Neurosurg 1982;56:430-434.

18. Voigt K, Yasargil G. Cerebral cavernous haemangiomas or cavernomas. Neurochirurgia(Stuttg) 1976;19:59-68.

19. Simard JM, Bengochea FG, Ballinger WE Jr, Mickle JP, Quisling RG. Cavernous angioma: a review of 126 collected and 12 new clinical cases. Neurosurgery 1986;18:162-172.

20. Berry RG, Alpers BJ, White JC. The site, structure and frequency of intracranial aneurysms, angiomas and arteriovenous abnormalities. In Millikans CH (ed). Research publications, Association for Research in Nervous and Mental Disease. Baltimore: Williams \& Wilkins, 1966:40-72.

21. Pozzati E, Padovani R, Morrone B, Finizio F, Gaist G. Cerebral cavernous angiomas in children. J Neurosurg 1980;53:826-832.
22. Sansone ME, Liwnicz BH, Mandybur TI. Giant pituitary cavernous hemangioma: case report. J Neurosurg 1980;53:124-126.

23. Khosla VK, Banerjee AK, Mathuriya SN, Mehta S. Giant cystic cavernoma in a child, case report. J Neurosurg 1984;60:1297-1299.

24. Chen TJ, Kuo TT. Giant intracranial Masson's hemangioma: report of a fatal case. Arch Pathol Lab Med 1984;108:555-556.

25. Dubovsky J, Zabramski JM, Kurth J, et al. A gene responsible for cavernous malformations of the brain maps to chromosome 7. Hum Molec Genet 1995;4:453-458.

26. Polymeropoulos $\mathrm{MH}$, Hurko $\mathrm{O}, \mathrm{Hsu} \mathrm{F}$, et al. Linkage of the locus for cerebral cavernous hemangiomas to human chromosome $7 \mathrm{q}$ in four families of Mexican-American descent. Neurology 1997;48:752-757.

27. Craig HD, Günel M, Cepeda O, et al. Multilocus linkage identifies two new loci for a Mendelian from stroke, cerebral cavernous malformation, at 7p15-13 and 3q25.2-27. Hum Molec Genet 1998;12:1851-1858.

28. Arnstein LH, Boldrey E, Nafziger HC. A case report and survey of brain tumors during the neonatal period. J Neurosurg 1951;8:315-319.

29. Thompson TP, Lunsford D, Flickinger JC. Radiosurgery for hemangiomas of the cavernous sinus and orbit: technical case report. Neurosurgery 2000;47:778-783. 